\title{
Ausmaß von Rationierung und Überversorgung in der stationären Versorgung
}

\author{
Ergebnisse einer bundesweiten Umfrage in deutschen Krankenhäusern
}

\author{
A. Reifferscheid ${ }^{1}$, N. Pomorin ${ }^{1}$, J. Wasem ${ }^{1}$
}

\section{Zusammenfassung}

Hintergrund und Fragestellung I Wirtschaftlicher Druck und eine unzureichende Investitionsförderung durch die Bundesländer führen bei vielen Krankenhäusern zu Verlusten. Weitgehend unklar ist, ob und inwieweit sich die Mittelknappheit in Form von Rationierung auf die Patientenversorgung auswirkt und welche Faktoren hierfür ursächlich sind. Auch wirft der Fallzahlanstieg die Frage auf, ob aus wirtschaftlichen Gründen zu viele medizinische Leistungen erbracht werden.

Methodik | Basierend auf früheren Studien sowie halbstrukturierten Interviews mit Leitungspersonen verschiedener Krankenhäuser wurden berufsgruppenspezifische Fragebögen entwickelt. Es wurden knapp 5000 Fragebögen an Chefärzte verschiedener Fachbereiche sowie Geschäftsführer und Pflegedirektoren verschickt. Der Rücklauf betrug insgesamt $43 \%$.
Ergebnisse I Von allen Befragten wurden deutliche finanzielle Restriktionen wahrgenommen. $46 \%$ der befragten Chefärzte gaben an, aus ökonomischen Gründen bereits nützliche Maßnahmen vorenthalten oder durch weniger effektive, aber kostengünstigere Alternativen ersetzt zu haben. Auch wenn Rationierung in allen Fachbereichen relativ verbreitet war, war die Intensität gering ausgeprägt. 39\% der Chefärzte glaubten, dass in ihrem Fachgebiet wirtschaftliche Rahmenbedingungen zu überhöhten Fallzahlen führen - insbesondere in der Orthopädie und der Kardiologie.

Schlussfolgerungen | Da sich die finanzielle Situation der Krankenhäuser mittelfristig kaum entspannen wird, besteht Handlungsbedarf für die Politik, um negative Auswirkungen auf die Patientenversorgung zu vermeiden.

\section{Einleitung}

Insbesondere innerhalb der Ärzteschaft wird eine zunehmende Ökonomisierung der Medizin kritisch diskutiert [1]. Im Krankenhaus (KH) haben die diagnosebezogenen-Fallgruppen-Pauschalen (DRG) maßgeblich zum Bedeutungszuwachs ökonomischer Aspekte beigetragen. Die Konstruktion des DRG-Systems ist dem ökonomisch-theoretischen Konzept der „yard stick competition“ zuzurechnen: Bei einem eingeschränkten Wettbewerb aufgrund staatlich reglementierter Preise sind alle Anbieter zu kontinuierlichen Effizienzsteigerungen angehalten [2]. Die Höhe der Fallpauschalen orientiert sich an den durchschnittlichen Behandlungskosten der jeweiligen Fallgruppe. $\mathrm{KH}$ mit einer ungünstigen Kostenstruktur verzeichnen Verluste, während Einrichtungen mit einem unterdurchschnittlichen Ressourceneinsatz Gewinne erzielen können. Die Einführung des DRGSystems und die damit verbundene Rationalisierung führten zu einer spürbaren Effizienzsteigerung [3].

Die Basisfallwerte sind in den letzten Jahren im Vergleich zu den Kostensteigerungen (insb. Gehälter, Energie, Versicherungsprämien) unterproportional angestiegen. Auch die unzureichende
Investitionsförderung durch die Bundesländer hat den ökonomischen Druck auf die KH erhöht. Der Investitionsstau im Krankenhaussektor wird gegenwärtig auf ca. 15 Mrd. € geschätzt [4]. Dies zwingt die KH implizit, Teile der für die Patientenversorgung gedachten Fallpauschalen für die Finanzierung von Investitionen zu verwenden.

Gegenwärtig verzeichnen 35-50\% der KH Verluste $[4,5]$. Bei vorgegebenen Preisen bleiben defizitären KH nur zwei Strategien, um Verluste zu kompensieren:

- die Kosten weiter zu senken oder

- die Fallzahlen zu erhöhen und die vorhandenen Kapazitäten stärker auszulasten.

Beide Strategien können theoretisch zu negativen Konsequenzen für die Patientenversorgung führen [6].

Kostenreduktion durch Rationalisierung, also das Ersetzen von Leistungen durch wirtschaftlichere Alternativen mit identischem Nutzen, ist nur begrenzt möglich. Sobald das Rationalisierungspotenzial weitgehend ausgeschöpft ist, erhöhen weitere Ausgabenreduktionen die Gefahr, dass erforderliche Leistungen durch weniger wirksame Maßnahmen ersetzt oder komplett unterlassen werden und sich so die Qualität der Patientenver-
Institute

1 Lehrstuhl für Medizinmanagement, Universität Duisburg-Essen
Korrespondenz Dipl.-Ges. Ök. Antonius Reifferscheid Lehrstuhl für Medizinmanagement, Fachbereich Wirtschaftswissenschaften Universität Duisburg-Essen, Campus Essen

Thea-Leymann-Straße 9 45127 Essen

Tel.: 0201-1833253

Fax: 0201-1833945

Antonius.Reifferscheid@ medman.uni-due.de 
sorgung verschlechtert. Der Literatur folgend wird die Vorenthaltung von nützlichen Maßnahmen bzw. deren Ersatz durch weniger effektive Leistungen als Rationierung verstanden - Rationierung ist somit die Vorenthaltung eines (Netto-)Nutzens für den Patienten [7, 8]. Da solche Rationierungsentscheidungen in Deutschland üblicherweise vom behandelnden Arzt getroffen werden, ohne dass er sich an expliziten für Dritte nachvollziehbaren Kriterien orientieren kann, hat sich der Begriff der impliziten Rationierung eingebürgert [7]. Außerdem wird von „nützlichen“ und nicht von „notwendigen“ Maßnahmen gesprochen, da die Notwendigkeit im Vergleich zum Nutzen als Konzept weniger eindeutig ist und subjektiv sehr unterschiedlich bewertet wird [8]. Die vorliegende Untersuchung fokussiert ausschließlich auf implizite Rationierung aus wirtschaftlichen Gründen. Die Vorenthaltung von Leistungen beispielsweise aus ethischen Erwägungen oder aufgrund von Fehlentscheidungen wird hingegen nicht berücksichtigt.

Zur Verbreitung der ökonomisch bedingten impliziten Rationierung in deutschen $\mathrm{KH}$ gibt es bislang nur zwei quantitative Forschungsarbeiten, die sich allerdings auf die Betrachtung medizinischer Hochkostenbereiche beschränken. Nach Boldt \& Schöllhorn gehen $67 \%$ der Intensivmediziner davon aus, dass Rationierung auf Intensivstationen stattfindet [9]. Bei Strech et al. gaben insgesamt 77\% der Intensivmediziner und Kardiologen an, innerhalb der letzten 6 Monate aus Kostengründen bestimmte Leistungen vorenthalten zu haben, während $13 \%$ der befragten Ärzte häufig (mind. einmal pro Woche) rationierten [7].

Auch die Fallzahlentwicklung kann Probleme für die Patientenversorgung mit sich bringen. Seit DRG-Einführung im Jahr 2004 sind die Fallzahlen

Tab. 1 Rücklauf Fragebogenversand nach Berufsgruppen und Fachabteilungen

\begin{tabular}{|llll|}
\hline Berufsgruppe & $\begin{array}{c}\text { Verschickte } \\
\text { Fragebögen }\end{array}$ & $\begin{array}{l}\text { Erhaltene } \\
\text { Antworten }\end{array}$ & Rücklaufquote \\
\hline CÄ & 3163 & 1432 & $45 \%$ \\
\hline Innere Medizin & 209 & - \\
\hline Allgemeine Chirurgie & 208 & - \\
\hline Gynäkologie/ Geburtshilfe & 200 & - \\
\hline Kardiologie & 117 & - \\
\hline Intensivmedizin & 218 & - \\
\hline Unfallchirurgie/Orthopädie & 379 & - \\
\hline Anästhesie ohne Intensivbetten & 63 & - \\
\hline Sonstige/ keine Angabe & 38 & - \\
\hline GF & 869 & 284 & $33 \%$ \\
\hline PD & 865 & 396 & $46 \%$ \\
\hline
\end{tabular}

bis 2012 um 1,8 Mio. bzw. 11\% gestiegen [10]. Eine denkbare Erklärung ist, dass die Vergütung pro Fall den Anreiz setzt, möglichst viele Patienten zu behandeln, insbesondere wenn die Vergütung je zusätzlichen Fall die Grenzkosten übersteigt. Die Konsequenz wäre, dass Patienten Leistungen erhalten, die sie nicht benötigen. Erhält ein Patient Leistungen, die keinen Nutzen erwarten lassen (sondern ggf. den Patienten direkt oder indirekt schädigen können), wird von Überversorgung gesprochen [11]. Im Rahmen dieser Arbeit ist ausschließlich die wahrgenommene Überversorgung aus wirtschaftlichen Gründen von Interesse. Andere Ursachen von Überversorgung (z.B. unzureichende Fachkenntnisse o. Ä.) werden nicht betrachtet.

Es sind auch Fallzahlsteigerungen aufgrund des demografischen Wandels sowie des medizinischen Fortschritts zu erwarten. Allerdings haben sich die Fallzahlen klar überproportional zur Alterung der Bevölkerung entwickelt [12]. Auch im internationalen Vergleich steigen in Deutschland - trotz eines hohen Ausgangsniveaus - die Fallzahlen stärker als in allen anderen Ländern [13]. Die Fallzahlen sind in der Kardiologie und der Orthopädie besonders stark gestiegen, was ein Indiz für eine ökonomisch-motivierte Überversorgung ist [12]. Zwar liefern verschiedene Arbeiten entsprechende Hinweise für einzelne Leistungsbereiche $[14,15]$ - ein breiter Überblick über die Problematik der stationären Überversorgung fehlt jedoch [11]. Angesichts dieser Forschungsdefizite bestand das Ziel der vorliegenden Arbeit darin, einen fachabteilungsübergreifenden Überblick über die von den Führungskräften wahrgenommene Versorgungssituation hinsichtlich Rationierung und Überversorgung im Klinikalltag zu geben.

\section{Methodik}

In einem mehrstufigen Forschungsdesign wurden zunächst berufsgruppenspezifische Interviewleitfäden entwickelt und 12 halbstrukturierte Interviews mit

- Chefärzten (CÄ),

- Geschäftsführern/kfm. Direktoren (GF) und

- Pflegedirektoren (PD) unterschiedlicher Krankenhausträger und Versorgungsstufen durchgeführt, transkribiert und mittels qualitativer Inhaltsanalyse ausgewertet [16]. Darauf aufbauend wurden spezifische Fragebögen für die CÄ, GF und PD entwickelt, welche die jeweiligen beruflichen Erfahrungsbereiche abbilden. Um einen hohen Rücklauf zu gewährleisten, wurden die Fragebögen auf zwei Seiten begrenzt. Soweit möglich wurden Fragen aus vergleichbaren Studien genutzt [u.a. 7, 9, 17].

Da das Begriffsverständnis der Befragten von Rationierung und Überversorgung unterschiedlich 


\begin{tabular}{|c|c|c|c|c|c|}
\hline Geschäftsergebnis 2013 & $\begin{array}{l}\text { negatives } \\
\text { Ergebnis }\end{array}$ & $\begin{array}{l}\text { ausgeglichenes } \\
\text { Ergebnis }\end{array}$ & $\begin{array}{l}\text { positives } \\
\text { Ergebnis }\end{array}$ & \multicolumn{2}{|c|}{ unbekannt/k.A. } \\
\hline CÄ & $41,6 \%$ & $20,0 \%$ & $35,3 \%$ & \multicolumn{2}{|l|}{$3,1 \%$} \\
\hline GF & $44,0 \%$ & $19,7 \%$ & $35,6 \%$ & \multicolumn{2}{|l|}{$0,7 \%$} \\
\hline PD & $47,5 \%$ & $19,4 \%$ & $29,3 \%$ & \multicolumn{2}{|l|}{$3,8 \%$} \\
\hline Krankenhausgröße & 100-250 Betten & 251-400 Betten & 401-550 Betten & >550 Betten & k.A. \\
\hline CÄ & $31,8 \%$ & $29,1 \%$ & $13,8 \%$ & $21,2 \%$ & $4,1 \%$ \\
\hline GF & $29,2 \%$ & $30,6 \%$ & $12,0 \%$ & $27,8 \%$ & $0,4 \%$ \\
\hline PD & $30,8 \%$ & $26,0 \%$ & $17,4 \%$ & $25,3 \%$ & $0,5 \%$ \\
\hline Statistisches Bundesamt* & $46,0 \%$ & $24,6 \%$ & $14,2 \%$ & \multicolumn{2}{|l|}{$15,2 \%$} \\
\hline Trägerschaft & Öffentlich & freigemeinnützig & privat & \multicolumn{2}{|l|}{ k. A. } \\
\hline CÄ & $40,4 \%$ & $40,3 \%$ & $19,1 \%$ & \multicolumn{2}{|l|}{$0,1 \%$} \\
\hline GF & $44,4 \%$ & $47,9 \%$ & $7,4 \%$ & \multicolumn{2}{|l|}{$0,4 \%$} \\
\hline PD & $38,4 \%$ & $49,0 \%$ & $11,1 \%$ & \multicolumn{2}{|l|}{$1,5 \%$} \\
\hline Statistisches Bundesamt* & $39,1 \%$ & $42,2 \%$ & $18,7 \%$ & & \\
\hline
\end{tabular}

Tab. 2 Repräsentativität nach Berufsgruppen

ausgeprägt sein könnte, wurde bei der Formulierung bewusst auf die explizite Nennung dieser Begriffe verzichtet [7, 17]. Um die Vergleichbarkeit mit früheren Studien weiterführend sicherzustellen, wurde Rationierung über folgende Frage erfasst: „Wie häufig haben Sie in den letzten 6 Monaten eine für den Patienten nützliche Maßnahme aus Kostengründen nicht durchgeführt bzw. durch eine preiswertere und zugleich weniger effektivere Leistung ersetzen müssen?" [ 7 , 17]. Zur ökonomisch motivierten Überversorgung existieren mangels früherer Studien [11] keine erprobten Instrumente. Da die Interviews vermuten ließen, dass Überversorgungstendenzen nicht direkt eingeräumt werden, wurde diesbezüglich indirekt formuliert und auf das eigene Fachgebiet bezogen gefragt: „Glauben Sie, dass die wirtschaftlichen Rahmenbedingungen in Ihrem Fachgebiet zu überhöhten Eingriffszahlen führen?" Es wurden jeweils vier- bis fünfstufigen Antwort-Skalen zum Ankreuzen vorgegeben.

Das Krankenhausverzeichnis und die Qualitätsberichte nutzend, wurde eine Kontaktdatenbank für den Fragebogenversand erstellt. Damit ein aussagekräftiger Überblick über die klinische Situation entstehen konnte, wurden - neben GF und PD CÄ zahlreicher Fachrichtungen

- Innere Medizin (Innere),

- Allgemeine Chirurgie (Allg. Ch.),

- Gynäkologie/Geburtshilfe (Gyn./Geb.),

- Kardiologie,

- Intensivmedizin (Intensiv) und Anästhesie (nicht bettenführend),

- Unfallchirurgie/Orthopädie (U.Ch./Ortho.) berücksichtigt. Die Studie wurde auf KH mit mehr als 100 Betten begrenzt, da die Führungsstrukturen in kleineren KH häufig nicht eindeutig differenziert sind (GF gleichzeitig CÄ oder kein PD vorhanden). Aus jedem Fachgebiet wurden 500 CÄ zufällig ausgewählt. In Fachgebieten mit insgesamt weniger als 500 Kontakten wurden sämtliche CÄ angeschrieben. Auf Erinnerungsschreiben oder finanzielle Anreize wurde verzichtet.

Neben einer deskriptiven Beschreibung der Antworten wurde die vorgefundene Rationierungsintensität auf Einflussfaktoren untersucht. Hierfür wurden ausschließlich die Antworten der CÄ genutzt, wozu aufgrund fehlender Angaben 1333 von 1432 Rückläufen verwendet werden konnten. Für die statistische Analyse wurde die Rationierungsintensität als binäre Variable kodiert (nie/ halbjährlich vs. monatlich/ wöchentlich) und mittels einer binär logistischen Regression in SPSS 20 auf potentielle Einflussfaktoren (ebenfalls auf binärem Messniveau) untersucht.

\section{Ergebnisse}

Deskriptive Statistik | Der Rücklauf betrug insgesamt $43 \%$ und fiel bei den CÄ und PD etwas höher aus als bei den GF (s. > Tab. 1).

Der Rücklauf war für die Grundgesamtheit (KH mit mehr als 100 Betten) in Bezug auf die finanzielle Situation der KH (gemäß Literatur verzeichnen $35-50 \%$ der KH Verluste $[4,5])$ und bei den CÄ bei der Trägerschaft weitgehend repräsentativ 


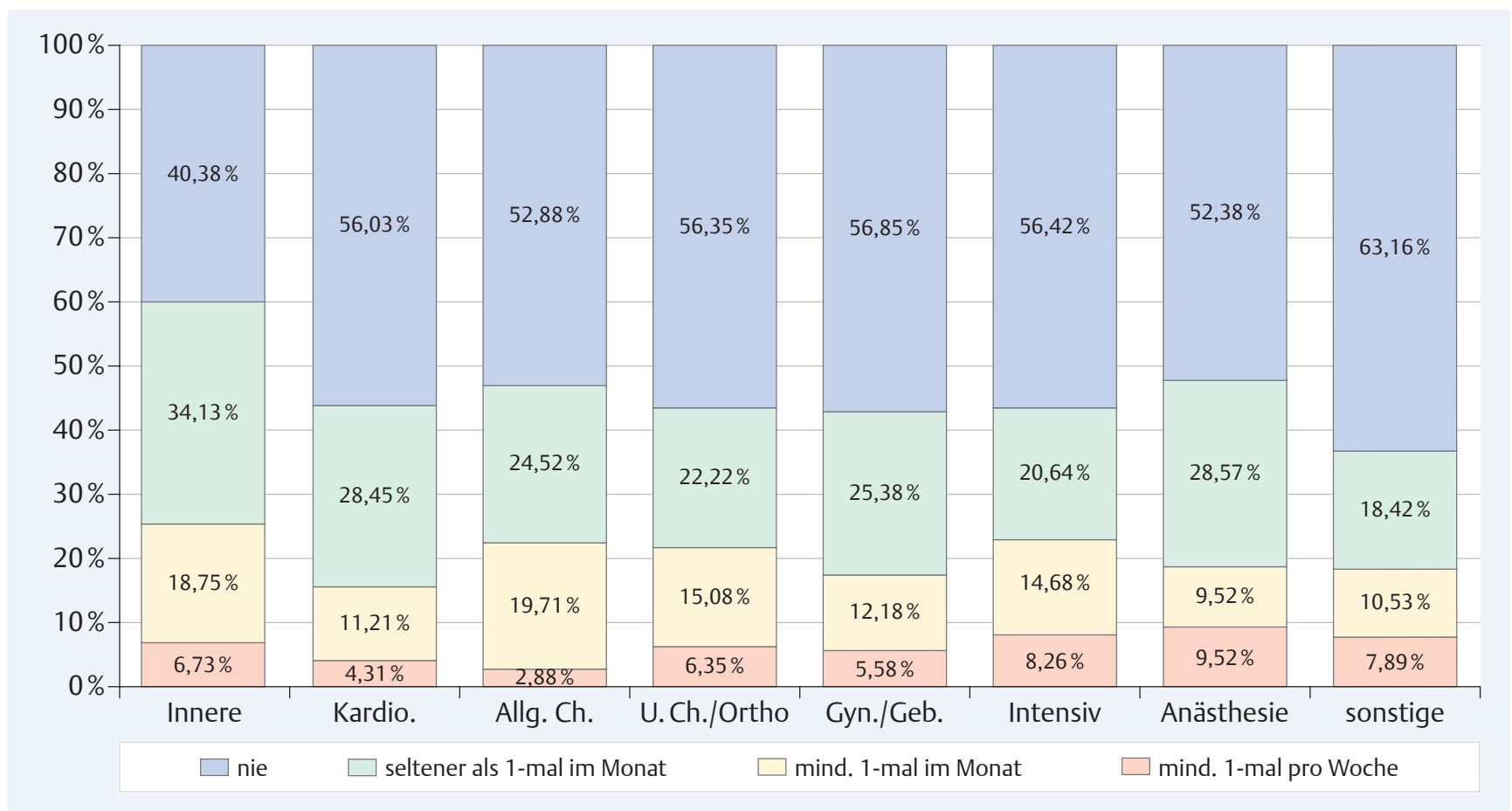

Abb. 1 Rationierungsintensität nach Fachabteilungen (s. > Tab. 2). Große KH sind tendenziell häufiger vertreten und bei den GF und PD sind private Träger etwas unterrepräsentiert.

Wahrnehmung der wirtschaftlichen Rahmenbedingungen I Der hohe wirtschaftliche Druck war für

- $81 \% \operatorname{der} C \ddot{A}$,

- $95 \%$ der GF sowie

- $93 \%$ der PD

deutlich spürbar. $45 \%$ der CÄ nahmen oft Entscheidungskonflikte zwischen ärztlichen und wirtschaftlichen Zielsetzungen wahr. 70\% der CÄ fühlten sich von den wirtschaftlichen Rahmenbedingungen in der Ausübung ihrer ärztlichen Tätigkeit eingeschränkt. Von 78\% wurden Beeinträchtigungen der Arbeitszufriedenheit aufgrund der wirtschaftlichen Rahmenbedingungen beklagt. Dabei zeigten sich kaum Unterschiede zwischen den Fachgebieten.

Die CÄ sahen nur wenig Raum für eine noch wirtschaftlichere Leistungserbringung. 70\% hielten das Rationalisierungspotenzial im ärztlichen Bereich für ausgeschöpft (bei den GF vertraten hingegen $61 \%$ die gegenteilige Meinung). Die allgemein schlechte wirtschaftliche Situation der $\mathrm{KH}$ und fehlende Möglichkeiten zur weiteren Kostenreduktion könnten Rationierung zur Folge haben. Entsprechend nahmen 50\% der CÄ und 43\% der GF wahr, dass aufgrund der ökonomischen Rahmenbedingungen in ihrem $\mathrm{KH}$ nicht alle nützlichen medizinischen Leistungen erbracht werden können.

Rationierung I Insgesamt gaben 46\% der CÄ an, dass sie in den letzten 6 Monaten nützliche medi- zinische Leistungen aus Kostengründen nicht durchgeführt bzw. durch eine preiswertere und zugleich weniger effektive Leistung ersetzt haben. Allerdings rationierten nur 6\% der CÄ wöchentlich und weitere $15 \%$ monatlich. Somit war Rationierung zwar nach Wahrnehmung der CÄ relativ weit verbreitet, allerdings - zumindest gegenwärtig - nicht allzu häufig. Es bestanden kaum Unterschiede zwischen den Fachabteilungen (FAB) (s. Abb. 1). GF und PD bewerteten die Problematik vergleichbar. Nur 32\% der GF und 16\% der PD gingen davon aus, dass im eigenen $\mathrm{KH}$ nie rationiert wird (13\% bzw. 14\% haben die Frage nicht beantwortet). Umgekehrt gingen aber auch nur 5\% der GF und 9\% der PD davon aus, dass es oft zu Rationierung kommt.

Medizinische Versorgungsunterschiede von Privatpatienten und Versicherten der gesetzlichen Krankenkassen sahen $28 \%$ der CÄ. Nur $44 \%$ der CÄ sahen sich bei der Entscheidung über die Verwendung knapper Ressourcen durch die GF ausreichend unterstützt - 90\% der GF sahen hingegen eine ausreichende Unterstützung der CÄ gewährleistet. Bei $21 \%$ der CÄ existierten (schriftliche) Standards zur Begrenzung teurer Maßnahmen. Diese Regelungen schienen aber nicht zwangsläufig zu Rationierung zu führen - so gaben $38 \%$ der CÄ mit solchen Vorgaben an, keine Leistungen zu rationieren.

Einflussfaktoren von Rationierung I Die Einflussfaktoren der Rationierungsintensität wurden mittels einer binär logistischen Regression untersucht (s. - Tab. 3). Auch wenn Nagelkerkes $\mathrm{R}^{2}$ mit 0,226 anzeigt, dass die Intensität nur zu einem begrenzten Teil durch die gewählten Variablen 
erklärt werden kann, waren doch mehrere Einflussfaktoren signifikant:

- Rationierung wurde signifikant seltener in großen KH wahrgenommen.

- Die Rationierungsintensität war bei einer guten technischen Ausstattung niedriger.

- Eine gute ärztliche oder pflegerische Personalausstattung zeigte hingegen keinen signifikanten Effekt.

Es gab keine signifikanten Unterschiede zwischen verschiedenen Trägerformen. Auch ein finanziell negatives Ergebnis des KH sowie die Zugehörigkeit zu einem Krankenhausverbund zeigten keine signifikanten Effekte. Die Existenz von (schriftlichen) Standards bzgl. des Einsatzes von kostenaufwändigen Maßnahmen führte erwartungsgemäß zu einer höheren Rationierungsintensität. Rationierung wurde dort signifikant häufiger wahrgenommen, wo von Entscheidungskonflikten zwischen ärztlichen und wirtschaftlichen Zielsetzungen berichtet wurde. Auch ein als ge- ring eingeschätzter Spielraum für weitere Rationalisierung führte signifikant öfter zur Wahrnehmung von Rationierung. Eine wahrgenommene Zeitknappheit bei der Betreuung der Patienten sowie eine als ausreichend empfundene Unterstützung der CÄ seitens der GF bei wirtschaftlichen Fragen zeigten keinen signifikanten Zusammenhang mit der Rationierungsintensität.

Überversorgung | Ein leistungsorientiertes Vergütungssystem bewirkt starke Anreize zur Erbringung möglichst vieler (komplexer) Leistungen. Die Studie liefert Hinweise für wahrgenommene Überversorgungstendenzen. Die Frage zur ökonomisch-motivierten Überversorgung wurde jedoch nur allgemein auf das jeweilige Fachgebiet und nicht speziell auf die eigene Abteilung bezogen. Daher kann hier keine weiterführende Untersuchung von Einflussfaktoren erfolgen. Die Wahrnehmung von Überversorgung differenziert nach FAB ist in $>$ Abb. 2 dargestellt.
Tab. 3 Ergebnisse der binär logistischen Regression

\begin{tabular}{|c|c|c|c|c|c|c|c|}
\hline \multirow[t]{2}{*}{ Einflussfaktoren } & \multirow[t]{2}{*}{$\begin{array}{l}\text { nicht } \\
\text { standardisierte } \\
\text { Koeffizienten }\end{array}$} & \multirow[t]{2}{*}{$\begin{array}{l}\text { Standard- } \\
\text { fehler }\end{array}$} & \multirow[t]{2}{*}{$\begin{array}{l}\text { Odds } \\
\text { Ratio }\end{array}$} & \multicolumn{2}{|c|}{$\begin{array}{l}95 \%-K o n f i d e n z- \\
\text { intervall für Odds } \\
\text { Ratio }\end{array}$} & \multirow[t]{2}{*}{ p-Wert } & \multirow[t]{2}{*}{$\begin{array}{l}\text { standardisierte } \\
\text { Koeffizienten }\end{array}$} \\
\hline & & & & $\begin{array}{l}\text { Unterer } \\
\text { Wert }\end{array}$ & $\begin{array}{l}\text { Oberer } \\
\text { Wert }\end{array}$ & & \\
\hline $\begin{array}{l}\text { großes KH mit }>400 \text { Betten } \\
(1=\text { ja; } 0=\text { nein })\end{array}$ & $-0,624$ & 0,170 & 0,536 & 0,384 & 0,748 & $<0,001^{*}$ & $-0,050$ \\
\hline $\begin{array}{l}\text { gute ärztliche Ausstattung der } \\
\text { FAB ( } 1=\text { ja; } 0=\text { nein) }\end{array}$ & $-0,346$ & 0,200 & 0,707 & 0,478 & 1,046 & 0,083 & $-0,026$ \\
\hline $\begin{array}{l}\text { gute pflegerische Ausstattung } \\
\text { der FAB ( } 1=\text { ja; } 0=\text { nein) }\end{array}$ & 0,256 & 0,229 & 1,292 & 0,824 & 2,024 & 0,264 & 0,017 \\
\hline $\begin{array}{l}\text { gute technische Ausstattung der } \\
\text { FAB ( } 1=\text { ja; } 0=\text { nein })\end{array}$ & $-0,541$ & 0,154 & 0,582 & 0,430 & 0,788 & $<0,001^{*}$ & $-0,045$ \\
\hline $\begin{array}{l}\text { Freigemeinnütziger Träger } \\
(1=\text { ja; } 0=\text { nein })\end{array}$ & 0,077 & 0,169 & 1,080 & 0,775 & 1,505 & 0,650 & 0,006 \\
\hline Privater Träger ( 1 = ja; 0 = nein) & 0,153 & 0,211 & 1,165 & 0,771 & 1,762 & 0,469 & 0,010 \\
\hline $\begin{array}{l}\text { defizitärer Jahresabschluss } 2013 \\
\text { des KH ( } 1 \text { = ja; 0= nein) }\end{array}$ & 0,079 & 0,152 & 1,082 & 0,803 & 1,459 & 0,605 & 0,007 \\
\hline $\begin{array}{l}\text { KH Teil eines Verbundes } \\
\text { ( } 1=\text { ja; } 0=\text { nein })\end{array}$ & $-0,221$ & 0,164 & 0,802 & 0,582 & 1,106 & 0,178 & $-0,017$ \\
\hline $\begin{array}{l}\text { Standards zur Begrenzung teurer } \\
\text { Maßnahmen ( } 1=\text { ja; } 0=\text { nein) }\end{array}$ & 0,832 & 0,166 & 2,297 & 1,658 & 3,182 & $<0,001^{*}$ & 0,057 \\
\hline $\begin{array}{l}\text { häufige Entscheidungskonflikte } \\
\text { ( } 1 \text { = ja; } 0 \text { = nein) }\end{array}$ & 1,298 & 0,159 & 3,660 & 2,678 & 5,003 & $<0,001^{*}$ & 0,108 \\
\hline $\begin{array}{l}\text { zu wenig Zeit für einzelne } \\
\text { Patienten ( } 1=\text { ja; } 0=\text { nein) }\end{array}$ & 0,264 & 0,153 & 1,302 & 0,964 & 1,759 & 0,086 & 0,022 \\
\hline $\begin{array}{l}\text { weitere Rationalisierungsmög- } \\
\text { lichkeiten in FAB ( } 1 \text { = ja; } 0 \text { = nein) }\end{array}$ & $-0,784$ & 0,184 & 0,456 & 0,319 & 0,654 & $<0,001^{*}$ & $-0,060$ \\
\hline $\begin{array}{l}\text { ausreichende Unterstützung } \\
\text { durch GF ( } 1=\text { ja; } 0=\text { nein) }\end{array}$ & $-0,244$ & 0,163 & 0,784 & 0,570 & 1,078 & 0,134 & $-0,020$ \\
\hline Konstante & $-1,497$ & 0,263 & 0,224 & & & $<0,001^{*}$ & \\
\hline
\end{tabular}


39\% der CÄ gingen davon aus, dass in ihrem Fachgebiet teilweise zu viele Patienten behandelt werden. CÄ aus den Bereichen Kardiologie und Orthopädie nahmen mit $61 \%$ bzw. $47 \%$ die Problematik insgesamt signifikant stärker wahr.

\section{Limitationen}

Die vorliegende Befragung erfasst nur subjektive Wahrnehmungen der Leitungspersonen. Unterschiede in der subjektiven Bewertung klinischer Situationen durch Leitungspersonal und nicht-leitende Mitarbeiter können bestehen. Vorarbeiten zeigen z.B. einen größeren Anteil der Befragten, die eine Rationierung verneinen, bei Führungspersonen als bei Nicht-Leitungspersonal [7]. Hinsichtlich der Validität der Frage nach Rationierung sei auf die Argumentation von Strech \& Marckmann [17] verwiesen. Die Überversorgungsfrage lässt keine direkte Einschätzung des Ausmaßes von Überversorgung zu. Vielmehr wurde gemessen, inwieweit die CÄ eine ökonomisch motivierte Überversorgung als Problem innerhalb ihrer Fachrichtung wahrnehmen. Die identifizierten Fachabteilungsunterschiede müssen überdies mit gewisser Vorsicht interpretiert werden. Durch die allgemeine Diskussion zur Fallzahlentwicklung könnte auch die Wahrnehmung der CÄ fachgruppenspezifisch geprägt sein. Weitere Untersuchungen sind daher wünschenswert.

\section{Diskussion}

Die Studie zeigt, dass sich in der Wahrnehmung der Führungskräfte im KH der wirtschaftliche
Druck auf die Patientenversorgung auswirkt. Die CÄ sahen sich durch finanzielle Restriktionen Entscheidungskonflikten ausgesetzt und in der Berufsausübung eingeschränkt. Der bisherige Befund der wissenschaftlichen Literatur, dass die Rationierung vor allem in Hochkostenbereichen stattfindet [7, 9], konnte nicht bestätigt werden. Vielmehr wurde in praktisch allen Fachbereichen gegenwärtig implizite Rationierung wahrgenommen. Die wahrgenommene Intensität der Rationierung ist im direkten Vergleich mit anderen Studien auf etwas niedrigerem Niveau [7]. Im Einklang mit vorigen Arbeiten konnte in dieser Studie kein signifikanter Effekt der Trägerschaft festgestellt werden [7]. Zum Ausmaß von Überversorgung gibt es bislang keine direkt vergleichbaren Arbeiten [11]. Nach dieser Studie nehmen CÄ eine wirtschaftlich motivierte Überversorgung als ein relevantes Problem wahr. Hier besteht weiterer Forschungsbedarf. Von besonderem Interesse wäre beispielsweise der Abgleich von S3-Leitlinien und tatsächlichen Behandlungsverläufen.

\section{Fazit}

Sofern nicht rechtzeitig geeignete Maßnahmen getroffen werden, werden sich die dargestellten Probleme weiter verschärfen. So führt der demografische Wandel und medizinisch-technischer Fortschritt zu einer steigenden Nachfrage, während gleichzeitig die Einnahmen der gesetzlichen Krankenversicherungen zurückgehen und die Schuldenbremse die öffentlichen Ausgaben deckelt.

Wenn die Vorenthaltung nützlicher Leistungen unter den derzeitigen wirtschaftlichen Rahmen-

Abb. 2 Wahrnehmung von überhöhten Fallzahlen im eigenen Fachgebiet

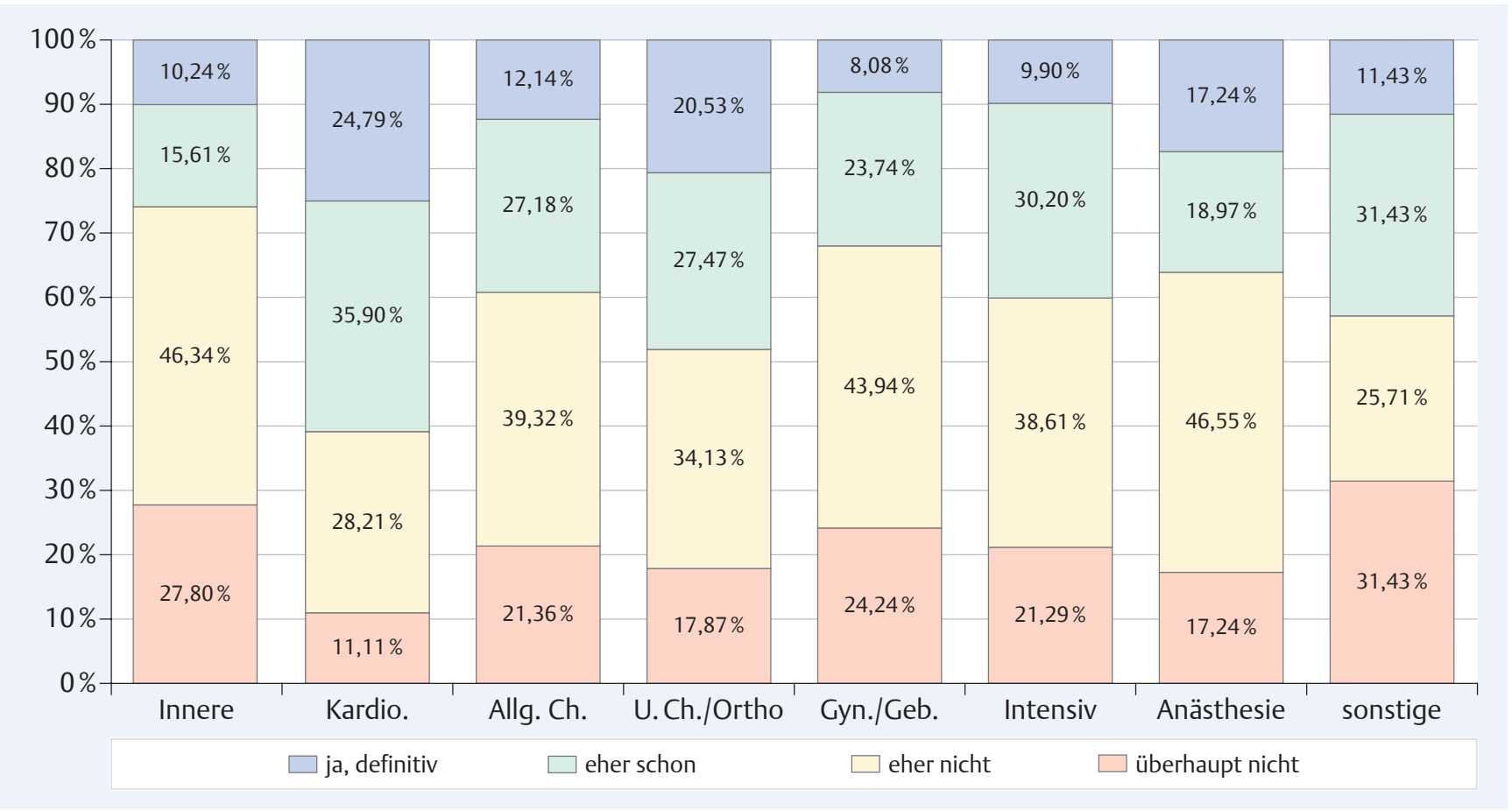


bedingungen unumgänglich ist, sollte die Entscheidung hierüber anhand definierter Kriterien erfolgen, um sowohl unerwünschte Wirkungen zu vermeiden, als auch um die Ärzteschaft in rechtlicher Hinsicht abzusichern. Folgerichtig sollte das Thema Priorisierung trotz fehlender Popularität auch auf politischer Ebene aufgegriffen werden. Der Forderung des Deutschen Ärztetages 2014 nach einer breiten gesellschaftlichen Debatte ist daher zuzustimmen.

Neben einer Definition prioritärer Leistungen könnte auch die im Entwurf des Versorgungsstärkungsgesetzes vorgesehene Ausweitung von Zweitmeinungsverfahren zum Abbau von Überversorgung beitragen. Angesichts des administrativen Aufwands erscheint es sinnvoll, sich auf auffällige Leistungsbereiche zu konzentrieren. Seit längerem wird ein Abbau von Überkapazitäten und eine Standortkonzentration gefordert (z.B. [13]). Andere Länder wie Dänemark und die Niederlande sind diesen Weg bereits gegangen und haben ihre stationären Kapazitäten reduziert und zentralisiert. Eine gezielte Konzentration der Krankenhausstrukturen erscheint jedoch politisch kaum durchsetzbar, sodass seitens der Politik weiter auf die wettbewerbliche Marktbereinigung gesetzt wird - mit entsprechenden Implikationen für die Patientenversorgung.

Zuletzt erscheint es dringend erforderlich, eine ausreichende Investitionskostenfinanzierung zu gewährleisten, um kurzfristig den wirtschaftlichen Druck auf die KH zu reduzieren und negative Konsequenzen für die Patientenversorgung abzuschwächen. Sofern die Bundesländer dazu alleine nicht in der Lage sind, müssen hier der Bund oder die Krankenkassen beteiligt werden.

\section{Konsequenzen für Klinik und Praxis}

- Rationierung ist keineswegs auf Hochkostenbereiche beschränkt, folglich sind in allen Fachgebieten Einschnitte in der Patientenversorgung zu befürchten.

- Die prognostizierte wirtschaftliche und morbiditätsbezogene Entwicklung wird die Rationierungsproblematik weiter verschärfen und die Gefahr einer unkontrollierten Mengendynamik erhöhen.

- Da implizite Rationierung auch mit rechtlichen Problemen verbunden ist, erscheint eine gesamtgesellschaftliche und vor allem gesundheitspolitische Debatte notwendig.

\section{Danksagung}

Unser Dank gilt den zahlreichen Teilnehmern der Befragung.

\section{Interessenkonflikt}

Das Projekt „Umgang mit Mittelknappheit im Krankenhaus", in dessen Rahmen diese Studie entstanden ist, wurde von der gemeinnützigen Dr. Jürgen Meyer Stiftung gefördert. Die inhaltliche wissenschaftliche Arbeit wurde durch die Förderung nicht beeinflusst.

\section{Literaturverzeichnis}

1 Hibbeler B. Ökonomisierung in der Medizin - Die Grenzen des Marktes. Deutsches Ärzteblatt 2013; 110: A1135-A1336

2 Street A, O’Reilly J, Ward P, Mason, A. DRG-based hospital payment and efficiency: Theory, evidence, and challenges. In: Busse R, Geissler A, Quentin W, Wiley M. Diagnosis-Related Groups in Germany. Berkshire: McGraw-Hill; 2011: 93-114

3 Tiemann O, Schreyögg J. Effects of ownership on hospital efficiency in Germany. Business Research 2009; 2: 115-145

4 Augurzky B, Hentschker C, Krolop S et al. Krankenhaus Rating Report 2014 - Mangelware Kapital: Wege aus der Investitionsfalle. Heidelberg: medhochzwei; 2014

5 Blum K, Löffert S, Offermanns M, Steffen P. Krankenhaus Barometer. Düsseldorf: Deutsches Krankenhaus Institut; 2013

6 Ellis R. Creaming, skimping and dumping: provider competition on the intensive and extensive margins. J Health Econ 1998; 17: 537-555

7 Strech D, Danis M, Löb M, Marckmann G. Ausmaß und Auswirkungen von Rationierung in deutschen Krankenhäusern - Ärztliche Einschätzungen aus einer repräsentativen Umfrage. Dtsch Med Wochenschr 2009; 134: 1261-1266

8 Breyer F. Implizite versus explizite Rationierung von Gesundheitsleistungen. Bundesgesundheitsblatt Gesundheitsforschung Gesundheitsschutz 2012; 55: 652-659

9 Boldt J, Schöllhorn T. Rationierung ist längst Realität - Ergebnisse einer Fragebogenaktion auf deutschen Intensivstationen. Deutsches Ärzteblatt 2008; 105: A995-A997

10 Statistisches Bundesamt. Grunddaten der Krankenhäuser - Fachserie 12 Reihe 6.1.1. Wiesbaden: Statistisches Bundesamt; 2013

11 Strech D. Der Abbau von Überversorgung als Teil der ärztlichen Berufsethik. Z Gerontol Geriatr 2014; 47: 17-22

12 Felder S, Mennicken R, Meyer S. Die Mengenentwicklung in der stationären Versorgung und Erklärungsansätze. In: Klauber J, Geraedts M, Friedrich J, Wasem J. Krankenhaus-Report 2013. Stuttgart: Schattauer; 2013: 95-109

13 OECD. Managing Hospital Volumes - Germany and Experiences from OECD Countries. Paris; 2013 http://docs.dpaq.de/3354-oecd_hospital_ volumes_germany.pdf Letzter Zugriff am 13.05.2015

14 Brause M, Grande G, Mannebach H, Badura B. Der Einfluss sozialer und struktureller Faktoren auf die Angemessenheit invasiver kardiologischer Prozeduren. Medizinische Klinik 2006; 101: 226-234

15 Dissmann W, de Ridder M. The soft science of German cardiology. Lancet 2002; 359: 2027-2029

16 Mayring P. Qualitative Inhaltsanalyse, Grundlagen und Techniken. 11th ed. Weinheim: Beltz; 2010

17 Strech D, Marckmann G. Wird in deutschen Kliniken rationiert oder nicht? Dtsch Med Wochenschr 2010; 135: 1498-1502

18 Statistisches Bundesamt. Krankenhausverzeichnis 2012. Wiesbaden: Statistisches Bundesamt; 2014
DOI 10.1055/s-0041-102550

Dtsch Med Wochenschr

2015; 140: e129-e135

(c) Georg Thieme Verlag KG . Stuttgart · New York . ISSN 0012-0472

\section{License terms}

